Nota

\title{
CAPACIDADE REPRODUTIVA DE Trichospilus diatraeae (HYMENOPTERA: EULOPHIDAE) EM PUPAS DE DIFERENTES HOSPEDEIROS (LEPIDOPTERA)
}

\author{
Miriam Rosa Paron ${ }^{1,3}$; Evôneo Berti-Filho* \\ ${ }^{1}$ Pós-Graduanda do Depto. de Entomologia, Fitopatologia e Zoologia Agrícola - USP/ESALQ. \\ ${ }^{2}$ Depto. de Entomologia, Fitopatologia e Zoologia Agrícola - USP/ESALQ, C.P. 9 - CEP: 13418-900 - Piracicaba, SP. \\ ${ }^{3}$ Bolsista CNPq. \\ *Autor correspondente <eberti@carpa.ciagri.usp.br>
}

RESUMO: Este trabalho teve como objetivo verificar a capacidade reprodutiva do parasitóide Trichospilus diatraeae Cherian \& Margabandhu, 1942 (Hym.: Eulophidae) em pupas de quatro hospedeiros lepidópteros: Diatraea saccharalis F. (Pyralidae), Anticarsia gemmatalis Hübner, Spodoptera frugiperda J.E. Smith e Heliothis virescens F. (Noctuidae). Os experimentos foram realizados em câmara climatizada do tipo B.O.D. a $25 \pm 1^{\circ} \mathrm{C}$, com $70 \pm 10 \%$ de Umidade Relativa e Fotofase de 14h. Não foi observada diferença na porcentagem de parasitismo entre as espécies, mas houve diferença no número de parasitóides emergidos, sendo menor em $H$. virescens (105,3 \pm 2,9 parasitóides/pupa) e maior em $S$. frugiperda (130,6 \pm 5,8 parasitóides/pupa). Todavia não houve diferença entre $D$. saccharalis (122,9 $\pm 5,5$ parasitóides/pupa) e $A$. gemmatalis (118,3 $\pm 6,5$ parasitóides /pupa). Palavras-chave: inseto, parasitóide, pupa

\section{REPRODUCTIVE CAPACITY OF Trichospilus diatraeae (HYMENOPTERA: EULOPHIDAE) ON DIFFERENT HOST PUPAE (LEPIDOPTERA)}

\begin{abstract}
This paper deals with the reproductive capacity of the endoparasitoid Trichospilus diatraeae Cherian \& Margabandhu, 1942 (Hym.: Eulophidae) on lepidopteran host pupae: Diatraea saccharalis F. (Pyralidae), Anticarsia gemmatalis Hübner, Spodoptera frugiperda J. E. Smith and Heliothis virescens Fabricius (Noctuidae). The experiments were carried out under control $\left(25 \pm 1^{\circ} \mathrm{C}, 70 \pm 10 \% \mathrm{RH}, 14\right.$-hour photofase). Parasitism did not differ significantly among host species. However there was significant difference in the progeny emergence, with the highest number for $S$. frugiperda (130.6 \pm 5.8 offsprings/pupa) and lowest for $H$. virescens (105.3 \pm 2.9 offsprings/pupa). However no significant difference between $D$. saccharalis (122.9 \pm 5.5 offsprings/pupa) and A. gemmatalis (118.3 \pm 6.5 offsprings/pupa) was observed.
\end{abstract}

Key words: insect, endoparasitoid, pupa

\section{INTRODUÇÃO}

Trichospilus diatraeae Cherian \& Margabandhu, 1942 (Hym., Eulophidae) originária da Ásia, é um parasitóide pupal preferencialmente da ordem Lepidoptera e tem sido estudado como agente potencial no controle biológico de pragas de importância da cana-de-açúcar, milho e algodão em países da África, Ásia e Américas (Boucek, 1976). No Brasil a ocorrência deste parasitóide é registrada pela primeira vez neste trabalho.

T. diatraeae é considerado um parasitóide polífago, sendo principalmente primário. O primeiro registro da ocorrência de $T$. diatraeae foi em 1942 no hospedeiro Diatraea venosata, a broca da haste de gramíneas. Entre 1963 a 1965, foi introduzido nas llhas Maurício e Reunião (Oceano Índico), onde foi registrado parasitando Chilo sacchariphagus sacchariphagus Bojer e Sesamia calamistis Hampson (Pyralidae). Na Índia e em Burma (Oriente Médio) foi registrado parasitando Hypsipyla robusta Moore, Sesamia inferens Walker, Sylepta derogata (F.), Pyrausta machaeralis Walker, Scirpophaga incertulas Walker e Chilo sacchariphagus indicus Kapur (Pyralidae) (Boucek, 1976).

Em 1963, T. diatraeae foi da Índia para Trinidad e Tobago (Antilhas) e para os EUA utilizando como hospedeiros Diatraea spp. (Pyralidae) (Bennett et al., 1987). Bordat et al. (1977) realizaram liberações de $T$. diatraeae para controle de pragas de gramíneas no Senegal (África), utilizando o hospedeiro Chilo suppressalis Walker (Pyralidae). Etienne \& Viette (1973) e Etienne (1975) relataram T. diatraeae, nas Ilhas Reunião e Maurício, parasitando as pragas da cana-de-açúcar Melanitis leda L. e Hedylepta indicata F. (Pyralidae) e Polydesma umbricola Boisduval (Noctuidae) praga da conífera Trichopolydesma collutrix. Bournier (1975) utilizou este parasitóide no controle biológico de noctuídeos pragas de algodão em Madagascar (África), utilizando como hospedeiro Ephestia (Anagasta) kuehniella Zell. Em 
Comores, Brenière et al. (1985) criaram e liberaram de $T$. diatraeae, na tentativa de controlar Chilo partellus Swinhoe (Pyralidae), praga do milho. Rodriguez-del-Bosque \& Smith Junior (1989) realizaram testes com T. diatraeae, nos EUA para controle da broca do milho Diatraea lineolata Walker (Pyralidae). Betbeder-Matibet (1990) estudou a criação massal de $C$. sacchariphagus para a obtenção de $T$. diatraeae. Torres-Bauza (1994) registrou o parasitóide atacando pupas de Dismorphia spio (Pieridae) em Inga vera (Mimosidae) em Porto Rico (Antilhas).

O objetivo deste trabalho foi avaliar a capacidade reprodutiva de $T$. diatraeae em quatro espécies de hospedeiros lepidópteros pragas: $D$. saccharalis $\mathrm{F}$. (Pyralidae), Anticarsia gemmatalis Hübner, Spodoptera frugiperda J.E. Smith e Heliothis virescens F. (Noctuidae).

\section{MATERIAL E MÉTODOS}

Os primeiros exemplares do parasitóide foram obtidos de uma pupa de Arctiidae coletada no campus da ESALQ em junho de 1996. Para a criação do parasitóide foi utilizada a metodologia descrita por Costa (1995) para parasitóides de pupários de mosca doméstica, com algumas modificações, como indicado a seguir. Foi utilizada uma sala de criação a $25 \pm 1^{\circ} \mathrm{C}$, fotofase de $14 \mathrm{~h}$ e sem controle da umidade relativa do ar. As gaiolas para a criação eram de madeira retangulares com $90 \times 50 \times 40 \mathrm{~cm}$; a parte superior com vidro transparente, as laterais $\mathrm{e}$ frontal fechadas com madeira, a posterior metade com madeira e metade com tela de náilon ("mesh" 0,3mm). Para a alimentação, utilizou-se algodão hidrófilo embebido em solução de mel (10\%), colocado em recipientes plásticos. Os hospedeiros utilizados para a criação foram pupas de lepidópteros com idade máxima de 3 dias. Três vezes por semana, estas pupas e a solução de mel eram substituídos por outros; as pupas parasitadas eram agitadas em uma peneira de malha 14 para separá-las das fêmeas do parasitóide. A seguir as pupas parasitadas eram colocadas individualmente em tubos de vidro de fundo chato (2,5 cm de diâmetro e $8,5 \mathrm{~cm}$ de comprimento), vedados com algodão hidrófobo envolto em tecido "voil", datados, acondicionados em bandejas plásticas e mantidos na sala de criação até a emergência dos parasitóides; à medida que emergiam, os parasitóides eram transferidos para as gaiolas de criação.

Para os experimentos foram utilizadas pupas de: $D$. saccharalis, $H$. virescens, $A$. gemmatalis e $S$. frugiperda obtidas de criação em dieta artificial. No caso de $D$. saccharalis, a maior parte de pupas foi obtida das criações dos laboratórios de Entomologia da COPERSUCAR (Cooperativa de Produtores de Cana, Açúcar e Álcool do Estado de São Paulo Ltda), Piracicaba, SP. Cada espécie de hospedeiro foi proveniente do mesmo lote de pupas, recém formadas e com peso semelhante.

Para a análise do parasitismo foi calculada a mortalidade corrigida pela fórmula de Abbott (1925), descontando-se a mortalidade natural do hospedeiro. $O$ delineamento estatístico utilizado para avaliar os parâmetros dos experimentos foi inteiramente casualizado. Os dados obtidos foram submetidos à análise de variância (SANEST) e as médias comparadas pelo teste de Tukey, ao nível de $5 \%$ de probabilidade.

\section{Três experimentos foram realizados:}

1) Cada espécie de hospedeiro foi exposta, individualmente, a uma fêmea do parasitóide recém emergida em um tubo de vidro (uma pupa de cada hospedeiro + uma fêmea do parasitóide) com 10 repetições. As pupas foram retiradas após 24 horas e a seguir individualizadas em tubos de vidro e colocadas na câmara climatizada a $25 \pm 1^{\circ} \mathrm{C}$, com $70 \pm 10 \%$ de umidade relativa e fotofase de 14 horas, até a emergência do parasitóide.

2) Os quatro hospedeiros (uma pupa de cada) foram expostos simultaneamente a uma fêmea do parasitóide e colocados em um tubo de vidro (quatro pupas de cada hospedeiro + uma fêmea do parasitóide) com 10 repetições. As pupas foram retiradas após um período de 24 horas, individualizadas em tubos de vidro e colocadas na câmara climatizada como descrito anteriormente.

3) Os quatro hospedeiros (5 pupas de cada) foram colocados em separado na gaiola de criação com parasitóides recém-emergidos ( 5 pupas de cada hospedeiro + várias fêmeas) e retirados após 24 horas; a seguir foram individualizados em tubos de vidro e colocados na câmara climatizada como descrito anteriormente.

Para avaliar a mortalidade natural dos hospedeiros, foram colocadas na mesma câmara climatizada utilizada em cada experimento 5 pupas de cada hospedeiro, não parasitadas.

\section{RESULTADOS E DISCUSSÃO}

Em nenhum dos três experimentos de $T$. diatraeae com os hospedeiros $S$. frugiperda, $A$. gemmatalis, $H$. virescens e $D$. saccharalis foi observada diferença significativa na porcentagem de parasitismo entre as espécies, mas houve diferença no número de parasitóides emergidos.

No experimento em que as pupas foram expostas individualmente a uma fêmea do parasitóide o número de parasitóides emergidos/pupa de $H$. virescens foi menor que o número de pupas de $S$. frugiperda e $A$. gemmatalis e semelhante ao número de parasitóides emergidos/pupa de D. saccharalis (TABELA 1).

No experimento em que as pupas das diferentes espécies de hospedeiros foram simultaneamente expostas a uma fêmea do parasitóide, houve diferença estatística entre $H$. virescens e as espécies $S$. frugiperda e $D$. saccharalis, em relação ao número de parasitóides emerigidos/pupa (TABELA 2). 
No experimento com exposição dos hospedeiros a muitas fêmeas do parasitóide houve parasitismo em quase $100 \%$ das pupas de todas as espécies de hospedeiro e o número de parasitóides emergidos/pupa de $S$. frugiperda foi maior que em pupas de $A$. gemmatalis e $H$. virescens (TABELA 3 ). O número de progênie do parasitóide em cada espécie de hospedeiro quase

TABELA 1 - Número de parasitóides emergidos/pupa e parasitismo de $T$. diatraeae sobre pupas de quatro espécies de hospedeiros expostos individualmente a uma fêmea do parasitóide. Temperatura $25 \pm 1,0^{\circ} \mathrm{C}$, Umidade Relativa $70 \pm 10 \%$ e Fotofase de 14 horas.

\begin{tabular}{lcc}
\hline Hospedeiro & $\begin{array}{c}\text { Parasitismo } \\
(\%)\end{array}$ & $\begin{array}{c}\text { Número de para sitóides } \\
\text { e mergidos/pupa }\end{array}$ \\
\hline $\begin{array}{l}\text { Spodoptera } \\
\text { frugiperda }\end{array}$ & $97,2 \pm 1,3 \mathrm{a}$ & $105,3 \pm 8,7 \mathrm{a}$ \\
$\begin{array}{l}\text { Diatraea } \\
\text { saccharalis }\end{array}$ & $96,4 \pm 2,2 \mathrm{a}$ & $97,5 \pm 6,2 \mathrm{ab}$ \\
$\begin{array}{l}\text { Anticarsia } \\
\text { gemmatalis } \\
\text { Heliothis } \\
\text { virescens }\end{array}$ & $97,1 \pm 1,4 \mathrm{a}$ & $103,7 \pm 10,4 \mathrm{a}$ \\
\hline
\end{tabular}

(1)Médias em cada coluna seguidas pela mesma letra não diferem pelo teste de Tukey a $5 \%$.

TABELA 2 - Número de parasitóides emergidos/pupa e parasitismo de T. diatraeae sobre pupas de quatro espécies de hospedeiros expostos simultaneamente a uma fêmea do parasitóide. Temperatura $25 \pm 1,0^{\circ} \mathrm{C}$, Umidade Relativa $70 \pm 10 \%$ e Fotofase 14 horas.

\begin{tabular}{lcc}
\hline Hospedeiro & $\begin{array}{c}\text { Parasitismo } \\
(\%)\end{array}$ & $\begin{array}{c}\text { Número de parasitó ides } \\
\text { emergidos/pupa }\end{array}$ \\
\hline $\begin{array}{l}\text { Spodoptera } \\
\text { frugiperda }\end{array}$ & $86,3 \pm 7,3 \mathrm{a}$ & $78,3 \pm 4,2 \mathrm{a}$ \\
$\begin{array}{l}\text { Diatraea } \\
\text { saccharalis }\end{array}$ & $85,3 \pm 4,3 \mathrm{a}$ & $76,5 \pm 3,5 \mathrm{a}$ \\
$\begin{array}{l}\text { Anticarsia } \\
\text { gemmatalis }\end{array}$ & $80,4 \pm 8,6 \mathrm{a}$ & $64,7 \pm 6,5 \mathrm{ab}$ \\
$\begin{array}{l}\text { Heliothis } \\
\text { virescens }\end{array}$ & $78,5 \pm 6,1 \mathrm{a}$ & $58,8 \pm 5,4 \mathrm{~b}$ \\
\hline
\end{tabular}

(1)Médias em cada coluna seguidas pela mesma letra não diferem pelo teste de Tukey a $5 \%$.

TABELA 3 - Número de parasitóides emergidos e parasitismo de $T$. diatraeae sobre pupas de quatro hospedeiros expostos individualmente a várias fêmeas do parasitóide. Temperatura $25 \pm 1,0^{\circ} \mathrm{C}$, Umidade Relativa $70 \pm 10 \%$ e Fotofase 14 horas.

\begin{tabular}{lcc}
\hline Hospedeiro & $\begin{array}{c}\text { Parasitismo } \\
(\%)\end{array}$ & $\begin{array}{c}\text { Número de parasitóides } \\
\text { e mergidos/pup } \mathrm{a}^{(1)}\end{array}$ \\
$\begin{array}{l}\text { Spodoptera } \\
\text { frugiperda }\end{array}$ & $98,8 \pm 0,8 \mathrm{a}$ & $208,3 \pm 4,5 \mathrm{a}$ \\
$\begin{array}{l}\text { Diatraea } \\
\text { saccharalis }\end{array}$ & $100,0 \pm 0,0 \mathrm{a}$ & $194,7 \pm 6,8 \mathrm{ab}$ \\
$\begin{array}{l}\text { Anticarsia } \\
\text { gemmatalis } \\
\text { Heliothis } \\
\text { virescens }\end{array}$ & $98,4 \pm 1,2 \mathrm{a}$ & $186,7 \pm 2,8 \mathrm{~b}$ \\
\hline
\end{tabular}

(1)Médias em cada coluna seguidas pela mesma letra não diferem pelo teste de Tukey a $5 \%$. duplicou em relação aos outros experimentos. Este maior número de progênie foi devido a oviposição de mais de uma fêmea no mesmo hospedeiro dentro da gaiola, acarretando superparasitismo (DeBach et al., 1991).

Nos três experimentos, apesar de não ter sido observada preferência do parasitóide às espécies de hospedeiro, observou-se que houve diferença no número de parasitóides emergidos/pupa entre os hospedeiros. Estas diferenças podem ser atribuídas à variação no tamanho entre os hospedeiros (as pupas maiores suportam o desenvolvimento de um maior número de imaturos), ou devido à resistência mecânica do tegumento das pupas, o que diminuiria a oviposição.

\section{CONCLUSÃO}

Todos os hospedeiros testados foram parasitados por $T$. diatraeae e houve emergência de progênie. Apesar de não ter sido observada preferência do parasitóide a uma das espécies de hospedeiro, comparando-se o número de parasitóides emergidos/pupa nas 4 espécies, observou-se que houve menor emergência em $\mathrm{H}$. virescens e maior em S. frugiperda.

\section{REFERÊNCIAS BIBLIOGRÁFICAS}

ABBOTT, W.S. A method of computing the effectiveness of an insecticide. Journal of Economic Entomology, v.18, p.265-267, 1925.

BENNETT, F.D.; GLENN, H.; YASEEN, M.; BARANOWSKI, R.M. Records of Trichospilus diatraeae, an Asian parasite (Hymenoptera: Eulophidae) from the Caribbean and Florida. Florida Entomologist, v.70, p.184-186, 1987.

BETBEDER-MATIBET, M. Élevage de plusieurs espéces du genre Chilo et de certains de leurs parasites pour la lutte biologique contre les foreurs des graminees en Afrique. Insect Science and Application, v.11, p.617-623, 1990.

BORDAT, D.; BRENIERE, J.; COQUARD, J. Foreurs de gramines africaines: parasitisme et techniques d'élevage. Agronomie Tropicale, v.32, p.391-399, 1977.

BOUCEK, Z. The African and Asiatic species of Trichospilus and Cotterelia (Hymenoptera, Eulophidae). Bulletin of Entomological Research, v.65, p.669-681, 1976.

BOURNIER, J.P. Sur la reproduction parthenogenetique de Trichosplilus diatraeae Cher. et Margab. (Hymenoptera: Chalcidoidea). Bulletin de la Societé Entomologique de France, v.80, p.116-118, 1975.

BRENIÈRE, J.; BORDAT, D.; VERCAMBRE, B.; HAMZA, H.; RENAND, M. Les opérations de lutte biologique contre le foreur du maïs Chilo partellus (Swinhoe), Lepidoptera, dans L'île de Ngazidja. Agronomie Tropicale, v.40, p.157-166, 1985.

CHERIAN, M.C.; MARGABANDHU, V. A new species of Trichospilus (Hymenoptera: Chalcidoidea) from south India. Indian Journal of Entomology, v.4, p.101-102, 1942.

COSTA, V.A. Efeito da temperatura na biologia de Spalangia gemina Boucek, 1963 (Hymenoptera, Pteromalidae), parasitóide pupal de Musca domestica L., 1758 (Diptera, Muscidae). Piracicaba, 1995. 67p. Tese (Doutorado) - Escola Superior de Agricultura "Luiz de Queiroz", Universidade de São Paulo. 
DeBACH, P.; ROSEN, D. Biological control by natural enemies. Cambridge: Cambridge University Press, 1991. 440p.

ÈTIENNE, J. Notes sur l'élevage et la biologie de Pseudoperichaeta laevis (Diptera: Tachinidae) sur hote de laboratoire. Entomophaga, v.20, p.105-111, 1975.

ÈTIENNE, J.; VIETTE, P. Nouvelle note sur Polydesma umbricola Boisduval (Lepidoptera: Noctuidae). Bulletin de la Société Entomologique de France, v.78, p.98-107, 1973.
RODRIGUEZ-DEL-BOSQUE, L.A.; SMITH JUNIOR, J.W. Parasitization of Diatraea lineolata pupa and diapausing larvae by several exotic parasites. Florida Entomologist, v.72, p.703-705, 1989.

TORRES-BAUZA, J.A. Hymenopterous parasitoids of Dismorfia spio (Pieridae: Dismorphiinae). Journal of the Lepidopterist's Society, v.48, p.266, 1994.

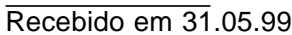

